This item was submitted to Loughborough's Research Repository by the author.

Items in Figshare are protected by copyright, with all rights reserved, unless otherwise indicated.

\title{
Vibration measurements using continuous scanning laser vibrometry: advanced aspects in rotor applications
}

PLEASE CITE THE PUBLISHED VERSION

PUBLISHER

(C) Elsevier

LICENCE

CC BY-NC-ND 4.0

REPOSITORY RECORD

Halkon, Ben J., and Steve Rothberg. 2019. "Vibration Measurements Using Continuous Scanning Laser Vibrometry: Advanced Aspects in Rotor Applications". figshare. https://hdl.handle.net/2134/3321. 
This item was submitted to Loughborough's Institutional Repository by the author and is made available under the following Creative Commons Licence conditions.

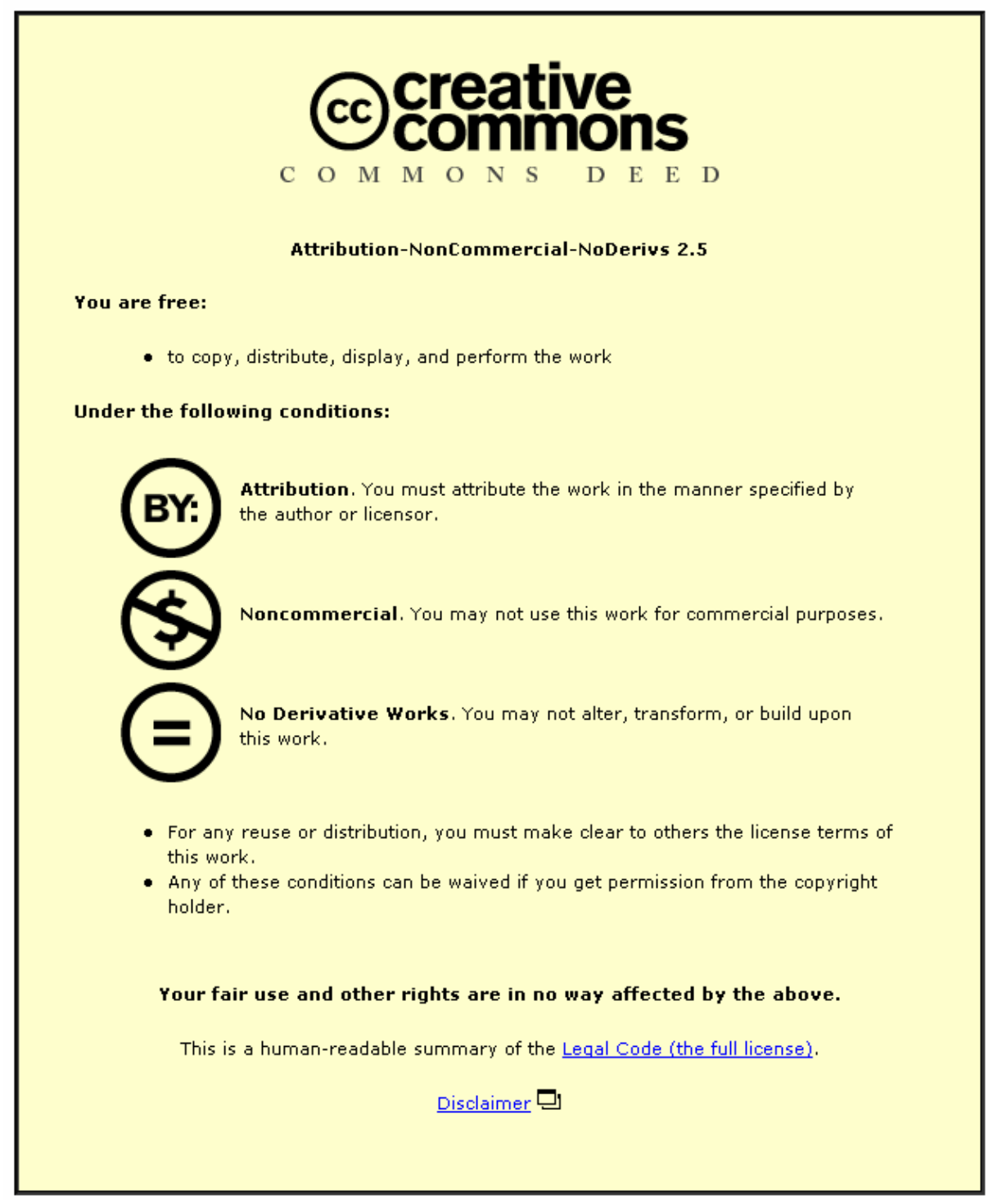

For the full text of this licence, please go to: http://creativecommons.org/licenses/by-nc-nd/2.5/ 


\section{VIBRATION MEASUREMENTS USING \\ CONTINUOUS SCANNING LASER VIBROMETRY: \\ ADVANCED ASPECTS IN ROTOR APPLICATIONS}

(CONTINUOUS SCANNING LASER VIBROMETRY:

ROTOR APPLICATIONS)

B. J. Halkon and S. J. Rothberg

Wolfson School of Mechanical and Manufacturing Engineering

Loughborough University, Loughborough, Leicestershire, LE11 3TU, U.K.

e-mail: b.j.halkon@lboro.ac.uk, tel: +44 (0) 1509 227589, fax: +44 (0) 1509227648 


\begin{abstract}
This paper builds on previous work concerned with the development of a comprehensive velocity sensitivity model for continuous scanning Laser Vibrometry. This versatile model predicts the measured velocity for arbitrary mirror scan angles and arbitrary target motion and it has been especially valuable in revealing the sources of additional components seen in continuous scanning and tracking measurements on rotors.

The application to vibration measurements on rotors is the particular focus of this paper which includes, for the first time, a three dimensional consideration of the incident point on the target and validation of the DC component of measured velocity leading to evaluation of the individual components of the small but inevitable misalignments between the rotor and optical axes. This has not previously been possible. Misalignments in the region $0.5 \mathrm{~mm}$ and $0.5^{\circ}$ were found and the model shows how additional components of the order $10-20 \mathrm{~mm} / \mathrm{s}$ result for typical measurements. Such levels are significant as they are comparable with vibration levels likely in real applications and, if unexpected, may lead to data misinterpretation.

The first thorough analysis of laser speckle effects in scanning Laser Vibrometer measurements on rotors is presented in the form of a speckle repeat map, together with experimental data quantifying the dramatic reduction in speckle noise found in tracking measurements. Finally, the velocity sensitivity model and the description of laser speckle effects are used to enable confident interpretation of data from a series of measurements on a rotating bladed disc.
\end{abstract}

KEYWORDS: Laser Doppler Vibrometry, scanning, tracking, vibration measurement, rotating machinery, laser speckle. 


\section{INTRODUCTION}

Laser Doppler Vibrometry (LDV) is now a well-established and commercially viable technique enabling non-contact vibration measurements in the most challenging of applications. Such instruments are technically well suited to general application but offer special benefits where certain constraints are imposed, for example by the context, which may demand high frequency operation, high spatial resolution or remote measurement, or by the structure itself, which may be hot, light or rotating. Measurements on such structures are often cited as important applications of LDV and scanning LDV is of particular current interest. Commercial scanning Laser Vibrometers incorporate two orthogonally aligned mirrors and can operate point by point, or in continuous scanning mode [1-4], a special case of which is the tracking condition in which the probe laser beam remains fixed on a single point on a moving target such as a rotating bladed disc [5]. Throughout the remainder of this paper, "scanning" LDV refers to operation in continuous scanning mode rather than point-by-point.

Recent work resulted in the extension $[3,4]$ of a totally general theoretical description of the velocity measured by a single laser beam incident in an arbitrary direction on a rotating target undergoing arbitrary motion [6] to the particularly challenging application of scanning Laser Vibrometer measurements on targets with flexible crosssections. The advanced technique of circular scanning on rotating targets was investigated as a means of illustrating the effectiveness of the resulting velocity sensitivity model for the analysis of actual scan configurations. In particular, the origins of the additional components that occur in measured data due to instrument configuration were easily revealed using the velocity sensitivity model.

This paper begins with a discussion of the theoretical aspects of circular scanning Laser Vibrometer measurements, defining a means of calculating the required mirror scan 
angles necessary to produce arbitrary scan profiles and an expression to predict measured velocity for any scan profile. The analysis then builds on the previously reported model to show how target shape, whilst distorting the scan profile, does not influence the measured velocity. The measured velocity is, however, affected by the scan configuration and the ability of the velocity sensitivity model to predict this effect and its experimental validation is a further focus of this paper.

A particularly important practical aspect of scanning LDV is associated with laser speckle induced noise and the resulting degradation of measured data. This paper contains the first detailed description of the characteristics of laser speckle noise in scanning measurements.

In the final section of the paper, a series of actual measurements are presented and analysed, the particular focus being the correct interpretation of the data obtained in a tracking Laser Vibrometer measurement on a rotating bladed disc. The information presented in the preceding sections is utilised, accurately predicting the form of resulting frequency spectra and enabling complex measurements such as this to be made with confidence.

\section{THEORETICAL ASPECTS OF SCANNING LASER VIBROMETRY}

\subsection{VELOCITY MEASURED BY A DUAL MIRROR SCANNING LASER}

\section{VIBROMETER}

With reference to Figure 1, a typical scanning measurement is performed by the introduction of two orthogonally aligned mirrors, separated by some distance $d_{S}$, into the laser beam path. 
In deriving the comprehensive velocity sensitivity model for scanning Laser Vibrometer measurements, it is possible to express the incident laser beam direction, $\hat{b}$, in terms of the mirror scan angles, $\theta_{S x}$ and $\theta_{S y}[3,4]$ :

$$
\hat{b}=\left[\sin 2 \theta_{S x}\right] \hat{x}-\left[\cos 2 \theta_{S x} \sin 2 \theta_{S y}\right] \hat{y}+\left[\cos 2 \theta_{S x} \cos 2 \theta_{S y}\right] \hat{z} .
$$

This important equation can be used to derive an expression for the velocity measured, $U_{m}$, in a scanning Laser Vibrometer measurement on a rotating target of flexible crosssection undergoing six degree-of-freedom vibration [3,4]:

$$
\begin{aligned}
U_{m} & =\sin 2 \theta_{S x}\left\lfloor\dot{x}_{r}\left(P_{0}\right)+\dot{x}_{f}(P)\right\rfloor \\
& -\cos 2 \theta_{S x} \sin 2 \theta_{S y}\left\lfloor\dot{y}_{r}\left(P_{0}\right)+\dot{y}_{f}(P)\right\rfloor \\
& +\cos 2 \theta_{S x} \cos 2 \theta_{S y}\left\lfloor\dot{z}_{r}\left(P_{0}\right)+\dot{z}_{f}(P)\right\rfloor,
\end{aligned}
$$

in which $\dot{x}_{f}(P), \dot{y}_{f}(P), \dot{z}_{f}(P)$ are the vibration velocity components in the $x, y, z$, directions due to cross-section flexibility (the point $P$ represents the instantaneous point of incidence of the laser beam on the arbitrarily deformed target) and $\dot{x}_{r}\left(P_{0}\right), \dot{y}_{r}\left(P_{0}\right)$, $\dot{z}_{r}\left(P_{0}\right)$ are the resultant vibration velocity components in the $x, y, z$ directions due to rigid body vibration (the point $P_{0}$ represents the corresponding point on the displaced but undeformed target), given by:

$$
\begin{aligned}
& \dot{x}_{r}\left(P_{0}\right)=\dot{x}-\left(\dot{\theta}_{z}+\Omega\right)\left(y_{0}-y\right)+\left(\dot{\theta}_{y}-\Omega \theta_{x}\right)\left(z_{0}-z\right), \\
& \dot{y}_{r}\left(P_{0}\right)=\dot{y}+\left(\dot{\theta}_{z}+\Omega\right)\left(x_{0}-d_{S} \tan 2 \theta_{S x}-x\right)-\left(\dot{\theta}_{x}+\Omega \theta_{y}\right)\left(z_{0}-z\right)
\end{aligned}
$$

and

$$
\dot{z}_{r}\left(P_{0}\right)=\dot{z}+\left(\dot{\theta}_{x}+\Omega \theta_{y}\right)\left(y_{0}-y\right)-\left(\dot{\theta}_{y}-\Omega \theta_{x}\right)\left(x_{0}-d_{S} \tan 2 \theta_{S x}-x\right)
$$

Here, $\dot{x}, \dot{y}, \dot{z}$ and $x, y, z$ are the rigid body translational vibration velocities and displacements, $\dot{\theta}_{x}, \dot{\theta}_{y}, \dot{\theta}_{z}$ and $\theta_{x}, \theta_{y}$ are the rigid body angular vibration velocities and displacements and $\Omega$ is the total rotation frequency of the target. $\left(x_{0}-d_{S} \tan 2 \theta_{S x}\right.$, 
$\left.y_{0}, z_{0}\right)$ describes the position of the laser beam incidence point on the $y$ deflection mirror - the most convenient "known point" along the line of the laser beam [3,4].

Equation (2) is important as it allows the user to predict the sensitivity of a scanning Laser Vibrometer measurement for any combination of mirror scan angles on any target. It readily accommodates the arbitrary time dependency in the mirror scan angles which is necessary to produce the scanning profiles that are discussed in this next section.

\subsection{PROFILE DETERMINATION FOR A DUAL MIRROR SCANNING LASER}

\section{VIBROMETER}

\subsubsection{Arbitrary Scan Profiles}

With reference to Figure 1, the position of the time dependent point of incidence of the laser beam on the arbitrarily shaped target, $\vec{r}_{P}$, can be described by $x_{S}(P), y_{S}(P)$ and $z_{S}(P)$ (omitting the explicit declaration of time dependency in $P$ for brevity in the equations):

$$
\vec{r}_{P}=\left[x_{S}(P)\right] \hat{x}+\left[y_{S}(P)\right] \hat{y}+\left[z_{S}(P)\right] \hat{z} .
$$

Consideration of the time dependent positions of the mirror incidence points and the target incidence point enables the quantities $x_{S}(P)$ and $y_{S}(P)$ to be re-expressed in terms of the time dependent mirror scan angles $\theta_{S x}$ and $\theta_{S y}$ [3]:

$$
x_{S}(P)=-\tan 2 \theta_{S x}\left(d_{S}+\frac{z_{0}-z_{S}(P)}{\cos 2 \theta_{S y}}\right)
$$

and

$$
y_{S}(P)=\left(z_{0}-z_{S}(P)\right) \tan 2 \theta_{S y} .
$$

In practice $z_{S}(P)$ is likely to be unknown and will thereby result in a difference between the desired and the actual beam incidence point. When $z_{S}(P)$ is small relative to $z_{0}$ such 
that it may be considered insignificant, equation (5b) can be rearranged such that the $y$ deflection mirror scan angle can be obtained for any $y_{S}(P)$. In either case, however, it can be seen from equation (5a) that $x_{S}(P)$ is never a simple function of the $x$ deflection mirror scan angle. This is particularly important when attempting to obtain a circular scan profile via the simultaneous modulation of the $x$ and $y$ deflection mirror scan angles as discussed in this next subsection.

\subsubsection{Circular Scan Profiles}

Typically "circular" scans are performed by using cosine and sine mirror drive signals of the form:

$$
\theta_{S x}=-\Theta_{S x} \cos \left(\Omega_{S} t+\phi_{S}\right)
$$

and

$$
\theta_{S y}=\Theta_{S y} \sin \left(\Omega_{S} t+\phi_{S}\right)
$$

where $\Theta_{S x}$ and $\Theta_{S y}$ are the $x$ and $y$ mirror scan amplitudes and $\Omega_{S}$ and $\phi_{S}$ are the scan angular frequency and initial phase. Accounting for the difference between the target to $x$ mirror and target to $y$ mirror distances, the use of mirror scan angles with unequal amplitudes results in an acceptable approximation to a circular scan profile $[3,4]$ :

$$
\Theta_{S x}=0.5 \tan ^{-1}\left(\frac{r_{S}}{z_{0}+d_{S}}\right)
$$

and

$$
\Theta_{S y}=0.5 \tan ^{-1}\left(\frac{r_{S}}{z_{0}}\right) .
$$

Substituting equations $(6 a \& b)$ and $(7 a \& b)$ into equations $(5 a \& b)$ enables the (small) difference between the scan profile that is obtained when employing such mirror scan angles and the desired circular profile to be observed [3,4]. The effect of any laser beam 
position error is clearly structure dependent but, in some cases, there may be a difference between the target velocity at the intended and actual measurement points.

Whilst the ability to calculate the required mirror scan angles necessary to produce specific scan profiles (and vice versa) is useful, particularly for more complex scans, the means to predict the measured velocity for any scan profile is especially important. This will be described with reference to circular scans in this next section.

\section{PRACTICAL ASPECTS OF SCANNING LASER VIBROMETRY: CIRCULAR SCANS}

Use of equation (2), the experimental validation of which will be discussed in this section, allows prediction of the measured velocity in a scanning Laser Vibrometer measurement. In particular it shows how additional components can occur when performing measurements on rotating targets. The prediction of such additional components in the Laser Vibrometer output demonstrates the value of the velocity sensitivity model very clearly - it enables the vibration engineer to predict the instrument output for any measurement configuration on any target and therefore make Laser Vibrometer measurements with confidence.

\subsection{ADDITIONAL COMPONENTS IN ROTATING TARGET MEASUREMENTS}

The prediction of the effects of translational and/or angular misalignment between the scanning system and target rotation axes on the measurement is particularly useful, since small misalignments are inevitable. Experience has shown that such small misalignments may lead to significant additional components in the velocity measured in a circular scanning measurement [4].

With reference to Figure 2, translational misalignment can be accounted for in the model by including the constants $x_{0 m}$ and $y_{0 m}$ in the known point $x$ and $y$ coordinates. 
Similarly, angular misalignment is represented by including the constants $\theta_{x m}$ and $\theta_{y m}$ in the angular vibration displacement parameters. Setting the flexible and rigid vibration components to zero in equation (2) enables the measured velocity (ideally zero, of course) to be predicted for this "no target vibration, arbitrary misalignment" case. Making use of equations (3a,b\&c), equation (2) can be used to show the influence of misalignments on the measured velocity:

$$
\begin{aligned}
& \frac{U_{m}}{\Omega}=\cos 2 \theta_{S x} \cos 2 \theta_{S y}\left[\theta_{x m} x_{0 m}+\theta_{y m} y_{0 m}\right] \\
& -\sin 2 \theta_{S x}\left\lfloor y_{0 m}+\theta_{x m}\left(z_{0}+d_{S} \cos 2 \theta_{S y}\right)\right\rfloor \\
& -\cos 2 \theta_{S x} \sin 2 \theta_{S y}\left\lfloor x_{0 m}-\theta_{y m} z_{0}\right\rfloor \\
& +d_{S} \sin 2 \theta_{S x} \sin 2 \theta_{S y} .
\end{aligned}
$$

Additional information exists in the measured Laser Vibrometer signal at integer multiples of the scan frequency, as illustrated in the simulated data in Figure 3 which incorporates typical values for a circular scan. Substitution for $\theta_{S x}$ and $\theta_{S y}$ using equations $(6 a \& b)$ and $(7 a \& b)$ and using small angle approximations enables this expression to be rewritten as:

$$
\begin{aligned}
\frac{U_{m}}{\Omega} & =\left[\theta_{x m} x_{0 m}+\theta_{y m} y_{0 m}\right] \\
& +\frac{r_{S}}{z_{0}+d_{S}}\left[y_{0 m}+\theta_{x m}\left(z_{0}+d_{S}\right)\right] \cos \left(\Omega_{S} t+\phi_{S}\right) \\
& -\frac{r_{S}}{z_{0}}\left[x_{0 m}-\theta_{y m} z_{0}\right] \sin \left(\Omega_{S} t+\phi_{S}\right) \\
& -\frac{d_{S} r_{S}^{2}}{2 z_{0}\left(z_{0}+d_{S}\right)} \sin 2\left(\Omega_{S} t+\phi_{S}\right),
\end{aligned}
$$

confirming that the most significant additional components occur at DC, $1 \mathrm{x}$ and $2 \mathrm{x}$ scan frequency. The additional components at DC (first set of terms in equation (9)) and $1 \mathrm{x}$ scan frequency (second and third sets of terms in equation (9)) are both dependent upon the misalignment. The $1 \mathrm{x}$ component is also dependent upon the scanning system configuration. The amplitude of the component at 2x scan frequency (fourth set of terms 
in equation (9)) is, however, insensitive to misalignments and, as such, it is possible to experimentally validate this element of the velocity sensitivity model separately.

\subsubsection{Dual Mirror Effects}

Since the amplitude of the $2 \mathrm{x}$ component is a function of the perpendicular mirror separation, $d_{S}$, as well as the scan radius, $r_{S}$, and the stand-off distance, $z_{0}$, the scanning system hardware used in this validation was designed with the facility to vary $d_{S}$ from $30 \mathrm{~mm}$ to $50 \mathrm{~mm}$. Experimental validation of the $2 \mathrm{x}$ component has been presented previously [4], confirming the dependency on $z_{0}$ and $r_{S}$, but Figure 4 shows new comparisons between the predicted and measured amplitude of the $2 \mathrm{x}$ component for a series of measurement configurations to substantiate this validation. The solid lines represent the theoretical prediction of $U_{m} / \Omega$ vs. $d_{S}$, with the plotted points representing the corresponding measured values. The theoretical prediction shows good correlation with the measured data.

The model can also be used to examine the effects of misalignment between the target and scanning system axes and this important aspect of real applications will be explored in detail in this next subsection.

\subsubsection{Misalignment Effects}

As shown in equation (9), translational and angular misalignments influence the DC and $1 \mathrm{x}$ additional measurement components. The custom built scanning system used here was also designed with the facility to vary $x_{0 m}, y_{0 m}$ and $\theta_{y m}$, such that the predicted and measured velocities could be compared for a series of misaligned configurations enabling validation of the velocity sensitivity model. In a previously presented experimental validation [4], only variations in the $1 \mathrm{x}$ component were considered but in this paper variations in the DC component are examined for this first time, ultimately 
enabling estimation of the individual initial misalignment parameters which has not previously been possible.

Whilst the "no target vibration" condition is relatively straightforward to achieve in the laboratory by taking care with target selection, the "no misalignment" condition is not. Small but inevitable initial misalignment between the scanning system and target rotation axes results in significant components at DC and 1x scan frequency.

Figure 5 shows comparisons between the predicted and measured amplitudes of the additional components at DC and 1x scan frequency for a series of measurements in which only the translational misalignment in the $x$ direction, $x_{0 m}$, was varied. Figure 6 shows similar comparisons in which only $y_{0 m}$ was varied. The broken lines represent the theoretical prediction of measured velocity per unit rotation frequency, $U_{m} / \Omega$, for varying $x_{0 m}$ or $y_{0 m}$ and the data points represent the corresponding series of measured values. The difference between the experimental data and the initial predictions in each set of data is significant and is due to the initial misalignments, which are unknown, difficult to control and cannot be measured directly. In the absence of a means to measure the initial misalignments directly, the chosen way to proceed is to use the velocity sensitivity model as a basis for a particular hypothesis. Experimentally demonstrating that the hypothesis holds can then be taken as validation of the model. The basis of this hypothesis is to use the velocity sensitivity model to obtain estimates for the initial misalignments after a series of measurements have been made in which one of the controllable misalignments is varied.

Showing the unknown initial misalignment parameters, $x_{0 m u}, y_{0 m u}, \theta_{x m u}$ and $\theta_{y m u}$, explicitly in equation (9) and evaluating at DC and 1x scan frequency results in:

$$
\frac{\left.U_{m}\right|_{\omega=0}}{\Omega}=\left(\theta_{x m}+\theta_{x m u}\right)\left(x_{0 m}+x_{0 m u}\right)+\left(\theta_{y m}+\theta_{y m u}\right)\left(y_{0 m}+y_{0 m u}\right)
$$


and

$$
\begin{aligned}
\left(\frac{\left.U_{m}\right|_{\omega=\Omega_{S}}}{\Omega}\right)^{2} & =\left(\frac{r_{S}}{z_{0}+d_{S}}\right)^{2}\left[\left(y_{0 m}+y_{0 m u}\right)+\left(\theta_{x m}+\theta_{x m u}\right)\left(z_{0}+d_{S}\right)\right]^{2} \\
& +\left(\frac{r_{S}}{z_{0}}\right)^{2}\left[\left(x_{0 m}+x_{0 m u}\right)-\left(\theta_{y m}+\theta_{y m u}\right) z_{0}\right]^{2} .
\end{aligned}
$$

Making $x_{0 m}$ the variable misalignment parameter, with $y_{0 m}, \theta_{x m}$ and $\theta_{y m}$ set to zero, enables these equations to be rearranged into forms that are useful for identifying the unknown misalignment parameters:

$$
\frac{\left.U_{m}\right|_{\omega=0}}{\Omega}=\theta_{x m u}\left(x_{0 m}+x_{0 m u}\right)+\theta_{y m u} y_{0 m u}
$$

and

$$
\begin{aligned}
\left(\frac{\left.U_{m}\right|_{\omega=\Omega_{S}}}{\Omega}\right)^{2}-\left(\frac{r_{S}}{z_{0}} x_{0 m}\right)^{2} & =r_{S}^{2}\left[\left(\frac{y_{0 m u}}{z_{0}+d_{S}}+\theta_{x m u}\right)^{2}+\left(\frac{x_{0 m u}}{z_{0}}-\theta_{y m u}\right)^{2}\right] \\
& +2\left(\frac{r_{S}}{z_{0}}\right)^{2} x_{0 m}\left(x_{0 m u}-z_{0} \theta_{y m u}\right) .
\end{aligned}
$$

The first part of the hypothesis is that plots of the terms on the left hand sides of these expressions against $x_{0 m}$ will result in good fits to straight lines and this is confirmed in Figure 5a for the DC component and Figure 7a for equation (11b) (the 1x component). Estimates of $\theta_{x m u}$ and $\left(x_{0 m u}-z_{0} \theta_{y m u}\right)$ based on the gradient of the plots in Figure 5a and Figure 7a, respectively, are then possible.

Following a similar procedure but, in this case, making $y_{0 m}$ the variable misalignment parameter, with $x_{0 m}, \theta_{x m}$ and $\theta_{y m}$ set to zero, should, again, result in plots that are good fits to straight lines. As illustrated in Figure $5 \mathrm{~b}$ and Figure $7 \mathrm{~b}$, this part of the hypothesis is further supported by the strength of these fits. In this case, the gradients of these plots can be used to obtain estimates of $\theta_{y m u}$ and $\left(y_{0 m u}+z_{0} \theta_{x m u}\right)$ and estimates of each of the individual initial misalignment parameters can be resolved. The angular misalignment 
parameters, $\theta_{x m}$ and $\theta_{y m}$, could also have been used in the same manner to obtain estimates of the unknown initial misalignments. The estimates of the individual unknown initial misalignments can then be used to update the theoretical prediction of the amplitude of the DC and 1x scan frequency components.

The second part of the hypothesis is that by substituting the estimated values of the unknown initial misalignment quantities into equations (10a\&b), much improved theoretical predictions of the DC and 1x scan frequency components will be obtained. These updated predicted amplitudes are also shown in Figure 5 and Figure 6 (solid lines) and they exhibit strong correlation with the measured data points, supporting this part of the hypothesis. The difference between the intercepts of the DC experimental and updated predicted amplitudes is due to a small DC drift on the dynamic signal analyser used.

The final part of the hypothesis is that estimates of the unknown initial misalignments should be reasonable given the care taken in ensuring that the scanning system and target rotation axes were aligned. In this experimental validation the initial angular misalignments, $\theta_{x m u}$ and $\theta_{y m u}$, are calculated as $-0.2^{\circ}$ and $0.7^{\circ}$, respectively, and the corresponding initial translational misalignments, $x_{0 m u}$ and $y_{0 m u}$, are calculated as $-0.38 \mathrm{~mm}$ and $-0.76 \mathrm{~mm}$. The nature of the experimental configuration used in this validation is such that these misalignments are quite reasonable, supporting the final part of the hypothesis. The strength of the hypothesis at each of the three stages is taken as a validation of the velocity sensitivity model for prediction of these significant DC and 1x scan frequency additional components. Use of the velocity sensitivity model for the estimation of the individual initial misalignment parameters is a significant new step and has been presented for the first time in this paper. 


\subsection{LASER SPECKLE EFFECTS}

The velocity sensitivity model, which expresses total target velocity in the direction of an arbitrarily orientated laser beam, does not include the effects of laser speckle, which are the result of an entirely different phenomenon. Since, however, an appreciation of laser speckle effects is so important in scanning LDV, they will be discussed at length in this section.

\subsubsection{Speckle Noise Formation}

Speckle patterns are formed whenever coherent light is scattered from a surface that is rough on the scale of the optical wavelength. Since this includes most surfaces of interest in engineering, the Doppler frequency shifted backscattered light collected in a typical Laser Vibrometer measurement will have the form of a speckle pattern. Speckle noise is introduced into the Laser Vibrometer output whenever the collected speckle pattern changes during the course of the measurement [7]. When the target or laser beam motion is such that the set of collected speckle patterns is recurrent in nature, the resulting speckle induced measurement noise has a pseudo-random form, characterised in the frequency domain by approximately equal amplitude peaks at the speckle pattern repeat frequency and higher order harmonics (low level broadband noise is also present in typical measured data).

Such speckle pattern changes will occur in a circular scanning measurement due to differences between the scan frequency and target rotation frequency, distortions in the scan trajectory and the target vibratory motion itself. Determination of the speckle repeat frequency can therefore be particularly complex and, if unexpected, may lead to data misinterpretation. 


\subsubsection{Speckle Noise Characteristics in Scanning Measurements on Rotating Targets}

A stationary laser beam incident on a rotating target will result in recurrent speckle noise which repeats at the target rotation frequency whilst a scanning laser beam incident on a stationary target will result in a speckle noise repeat at the scan frequency. A scanning laser beam incident on a rotating target can give rise to speckle noise which repeats at a frequency other than the scan or the rotation frequencies. In such a case, the speckle repeat has a period that corresponds to whenever both the scan and the rotation have completed integer numbers of cycles.

The velocity measured in such a measurement is illustrated in Figure 8 where a scan at $12.5 \mathrm{~Hz}$ combined with rotation at $10 \mathrm{~Hz}$ resulted in a speckle noise repeat at $2.5 \mathrm{~Hz}$, i.e. 5 cycles of scan and 4 cycles of rotation. The sharpness of these harmonic peaks and the high order up to which they prevail are classic characteristics of speckle noise [7].

This is, of course, just one of the many speckle repeat possibilities and a full map of speckle repeat frequencies is shown in Figure 9. The lower limit apparent on the ratio of speckle repeat frequency / rotation frequency is equal to the resolution in the spectrum. In this data this has been set at 1/50th of the rotation frequency, i.e. the data length is equal to the time taken for 50 rotation cycles. The solid line shown corresponds to four times the resolution in the spectrum and, based on experience, is proposed as the lowest speckle repeat frequency that could be seen clearly in the spectrum. The plot is dimensionless such that the LDV user could plot different limits on this particular map for any resolution coarser than 1/50th of the rotation frequency. The data points above the solid line thus represent the repeat frequencies that can be seen at all the specific values of the ratio of scan frequency / rotation frequency at which repeats could be observed. These must obviously include the example shown previously in Figure 8 and this data-point is shown highlighted in a circle in Figure 9. 
The tracking condition, where scan frequency / rotation frequency $=1$, merits further discussion. The map shows that the speckle repeat frequency should equal rotation frequency at this condition and, if perfect tracking could be achieved, the speckle pattern on the detector might be expected to rotate but not to change its form, resulting in extremely low noise in the instrument output. In reality, as discussed earlier, there are small but inevitable misalignments between target rotation and optical axes, as well as imperfections in the scan profile, that mean there will still be modest changes in the collected speckle pattern. Nonetheless, a significant drop in speckle noise does result as the tracking condition is approached and this is illustrated in Figure 10. Here, a more focused range $\left(0.8 \Omega \leq \Omega_{S} \leq 1.2 \Omega\right)$ than previously presented $\left(0 \leq \Omega_{S} \leq 2 \Omega\right)$ [4] is examined such that the effects close to tracking can be identified. Here, the spectral mean squared noise when tracking is at least a factor of 2 down on that when the scan and rotation frequencies differ by just a few percent.

\section{ROTOR VIBRATION MEASUREMENTS}

The main objective of this paper so far has been to validate the velocity sensitivity model for scanning measurements, in particular to verify the prediction of additional components that occur at integer multiples of the scan frequency in measurements on rotating targets. In this section, the velocity sensitivity model will be used to predict the form of the Laser Vibrometer output in a circular tracking measurement on a rotating axially flexible bladed disc undergoing a medium severity vibration. The vibration is generated by mounting the rotor assembly on a linear bearing and exciting it with an electrodynamic shaker.

Figure 11 shows the velocity measured in a Laser Vibrometer measurement on a nonrotating blade undergoing first natural frequency vibration at $32.5 \mathrm{~Hz}$ (nominal). This 
straightforward measurement constitutes a baseline for the tracking measurements, illustrating the vibration peak at $32.5 \mathrm{~Hz}$, as well as the genuine low-level harmonic distortions at $65 \mathrm{~Hz}$ and $97.5 \mathrm{~Hz}$. The underlying instrument noise floor contains peaks at $50 \mathrm{~Hz}$ and $100 \mathrm{~Hz}$, which are caused by mains electrical interference and, as such, are present in all measured spectra presented in this section.

Figure 12 shows the velocity measured in a tracking Laser Vibrometer measurement on a (nominally) non-vibrating, rotating blade. Here, the significantly higher velocity levels at $1 \mathrm{x}(20 \mathrm{~mm} / \mathrm{s})$ and $2 \mathrm{x}(13 \mathrm{~mm} / \mathrm{s})$ scan frequency, due to the arbitrary initial misalignment and the dual mirror configuration, respectively, are easily identifiable. These amplitudes are comparable with those shown earlier in Figure 3. In addition to this, the misalignments result in relative motion between the laser beam and blade surface causing speckle pattern motions with a repeat frequency equal to the rotation frequency. Despite the absence of a controlled excitation, the rotation of the target leads to measurable blade motion at frequencies close to resonance and this genuine velocity content is present in the data.

In the case of a tracking Laser Vibrometer measurement on a vibrating, rotating blade, illustrated in Figure 13, the measurable blade motion is evident at $32.5 \mathrm{~Hz}$ due to the controlled excitation (genuinely lower amplitude motion than in Figure 11 for the same excitation) and surrounding frequencies due to the rotation. As expected, there is additional measurement content at $1 \mathrm{x}$ and $2 \mathrm{x}$ scan frequency and speckle induced measurement noise for reasons detailed in this paper.

The theory presented in sections 2 and 3 enables the user to predict the form of the measured velocity and such a prediction is shown in Figure 14 for this particular tracking measurement arrangement with estimated misalignment values based on the measurement configuration. As can be seen by comparison of Figure 13 and Figure 14, 
an order of magnitude prediction is possible for the misalignment dependent DC and 1x components (DC not shown) whilst the $2 \mathrm{x}$ component can be predicted with a high degree of accuracy since it is insensitive to misalignment. The low-level component at 3x scan frequency in Figure 14 is also associated with misalignment [4] but is generally below the real instrument noise floor and therefore insignificant. The overall strong correlation for the three principal velocity components of significant amplitude demonstrates the usefulness of the velocity sensitivity model for such advanced LDV applications.

In the measurement discussed in this section, the rotation frequency and vibration level and frequency were chosen such that the additional measurement content did not constitute a significant source of measurement ambiguity. In a real measurement such control is obviously not available and, for example, if the axial vibration level were lower or at a different frequency, it may be less straightforward to distinguish genuine vibration peaks from additional content peaks.

\section{CONCLUSIONS}

The use of Laser Vibrometers incorporating some form of manipulation of the laser beam orientation, typically using two orthogonally aligned mirrors, has become increasingly popular in recent years. Considerable attention has been given to the operation of such scanning Laser Vibrometers in continuous scanning mode in which the laser beam orientation is a continuous function of time, making it possible, for example, to track a single point on a moving target such as a rotor. This paper has investigated the application of a previously developed velocity sensitivity model to this particularly challenging measurement. 
The revised velocity sensitivity model has been applied in this paper to show how additional components occur at DC and integer multiples of the scan frequency due to the dual mirror arrangement and misalignment between the scanning system and target rotation axes. In particular, the velocity sensitivity model has been used to calculate the individual misalignment parameters for the first time. The experimental validation of the model for the prediction of the amplitudes of these additional components was presented, further confirming its usefulness. The influence of laser speckle on measured data was highlighted as an important practical aspect of scanning LDV and a detailed discussion of the typical characteristics of laser speckle noise in scanning measurements on rotating target was presented for the first time.

Finally, the output from a real circular tracking measurement was interpreted correctly by making use of the information presented in this paper. The information presented in this paper provides the user with the ability to predict the additional components that occur in real scanning Laser Vibrometer measurements and thereby anticipate the form of the resulting spectra. Such measurements can then be interpreted with confidence. Such measurements would enable the acquisition of valuable data that would be extremely difficult to obtain by any other means, for the analysis of important effects such as centrifugal stiffening.

\section{ACKNOWLEDGEMENT}

The authors would like to acknowledge the support of the Engineering and Physical Sciences Research Council who are funding this project. 


\section{REFERENCES}

1. P. SRIRAM, J. I. CRAIG and S. HANAGUD 1990 The International Journal of Analytical and Experimental Modal Analysis 5(3), 155-167. A scanning laser Doppler vibrometer for modal testing.

2. A. B. STANBRIDGE and D. J. EWINS 1999 Mechanical Systems and Signal Processing 13(2), 255-270. Modal testing using a scanning laser Doppler vibrometer.

3. B. J. HALKON and S. J. ROTHBERG 2003 Measurement Science and Technology 14(3), 382-393. Vibration measurements using continuous scanning laser vibrometry: theoretical velocity sensitivity analysis with applications.

4. B. J. HALKON, S. R. FRIZELL and S. J. ROTHBERG 2003 Measurement Science and Technology 14(6), 773-783. Vibration measurements using continuous scanning laser vibrometry: velocity sensitivity model experimental validation.

5. P. CASTELLINI and N. PAONE 2000 Review of Scientific Instruments 71(12), 4639-4647. Development of the tracking laser vibrometer: performance and uncertainty analysis.

6. J. R. BELL and S. J. ROTHBERG 2000 Journal of Sound and Vibration 237(2), 245-261. Laser vibrometers and contacting transducers, target rotation and six degreeof-freedom vibration: what do we really measure?

7. S. J. ROTHBERG, J. R. BAKER and N. A. HALLIWELL 1989 Journal of Sound and Vibration 135(3), 516-522. Laser vibrometry: pseudo-vibrations. 


\section{LIST OF FIGURES}

Figure 1 - The dual mirror scanning arrangement

Figure 2 - Translational and angular misalignment between the scanning system and target rotation axes

Figure 3 - Additional measurement components that occur due to misalignment between the dual mirror scanning system and target rotation axes $\left(r_{S}=100 \mathrm{~mm}, d_{S}=\right.$ $50 \mathrm{~mm}, z_{0}=1 \mathrm{~m}, x_{0 m}=y_{0 m}=2 \mathrm{~mm}$ and $\left.\theta_{x m}=\theta_{y m}=15 \mathrm{mrad}\right)$

Figure 4 - Experimental validation of the additional measurement component at $2 \mathrm{x}$ scan frequency for $z_{0}=0.512 \mathrm{~m}$ (a) and $z_{0}=1.127 \mathrm{~m} \mathrm{(b)}\left(\Omega_{S}=40 \pi \mathrm{rad} / \mathrm{s}\right)$

Figure 5 - Experimental validation of the additional measurement component at DC (a) and $1 \mathrm{x}$ scan frequency (b) for varying $x_{0 m}\left(r_{S}=15 \mathrm{~mm}, d_{S}=50 \mathrm{~mm}, z_{0}=0.5 \mathrm{~m}, \Omega_{S}=40 \pi\right.$ $\mathrm{rad} / \mathrm{s}, y_{0 m}=0 \mathrm{~mm}$ and $\left.\theta_{x m}=\theta_{y m}=0 \mathrm{mrad}\right)$, broken line $=$ initial prediction, solid line $=$ updated prediction

Figure 6 - Experimental validation of the additional measurement component at DC (a) and $1 \mathrm{x}$ scan frequency (b) for varying $y_{0 m}\left(r_{S}=15 \mathrm{~mm}, d_{S}=50 \mathrm{~mm}, z_{0}=0.5 \mathrm{~m}, \Omega_{S}=40 \pi\right.$ $\mathrm{rad} / \mathrm{s}, y_{0 m}=0 \mathrm{~mm}$ and $\left.\theta_{x m}=\theta_{y m}=0 \mathrm{mrad}\right)$, broken line $=$ initial prediction, solid line $=$ updated prediction

Figure 7 - Initial unknown misalignment analysis using variable $x_{0 m}$ (a) and variable $y_{0 m}$ (b)

Figure 8 - Velocity measured by a circular scanning Laser Vibrometer on a rotating, non-vibrating target $\left(r_{S}=50 \mathrm{~mm}, d_{S}=50 \mathrm{~mm}, z_{0}=250 \mathrm{~mm}, \Omega_{S}=25 \mathrm{rad} / \mathrm{s}, \Omega \approx 20 \pi \mathrm{rad} / \mathrm{s}\right.$, arbitrary misalignment)

Figure 9 - Speckle repeat map 
Figure 10 - Speckle noise in circular scanning Laser Vibrometer measurements on rotating targets

Figure 11 - Velocity measured by a Laser Vibrometer on a non-rotating blade undergoing $32.5 \mathrm{~Hz}, 100 \mathrm{~mm} / \mathrm{s}$ (nominal) vibration

Figure 12 - Velocity measured by a circular tracking Laser Vibrometer on a (nominally) non-vibrating, rotating blade $\left(r_{S}=100 \mathrm{~mm}, d_{S}=50 \mathrm{~mm}, z_{0}=1 \mathrm{~m}, \Omega_{S}=\Omega \approx\right.$ $20 \pi \mathrm{rad} / \mathrm{s}$, arbitrary misalignment)

Figure 13 - Velocity measured by a circular tracking Laser Vibrometer on a rotating blade undergoing $32.5 \mathrm{~Hz}, 10 \mathrm{~mm} / \mathrm{s}$ (nominal) vibration $\left(r_{S}=100 \mathrm{~mm}, d_{S}=50 \mathrm{~mm}, z_{0}=\right.$ $1 \mathrm{~m}, \Omega_{S}=\Omega \approx 20 \pi \mathrm{rad} / \mathrm{s}$, arbitrary misalignment)

Figure 14 - Theoretical prediction of the velocity measured by a circular tracking Laser Vibrometer on a rotating target undergoing $32.5 \mathrm{~Hz}, 10 \mathrm{~mm} / \mathrm{s}$ vibration $\left(r_{S}=100 \mathrm{~mm}, d_{S}\right.$ $=50 \mathrm{~mm}, z_{0}=1 \mathrm{~m}, \Omega_{S}=\Omega=20 \pi \mathrm{rad} / \mathrm{s}, x_{0 m}=y_{0 m}=2 \mathrm{~mm}$ and $\left.\theta_{x m}=\theta_{y m}=5 \mathrm{mrad}\right)$ 


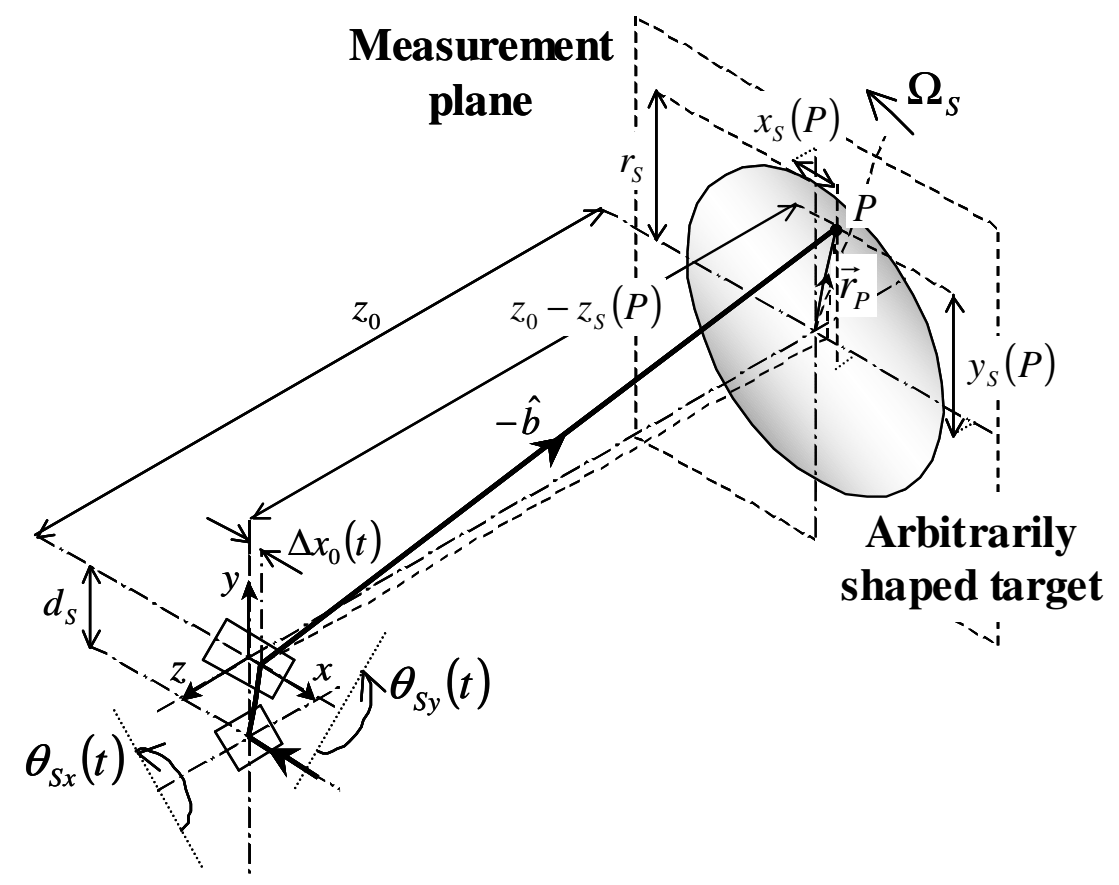

Figure 1 - The dual mirror scanning arrangement
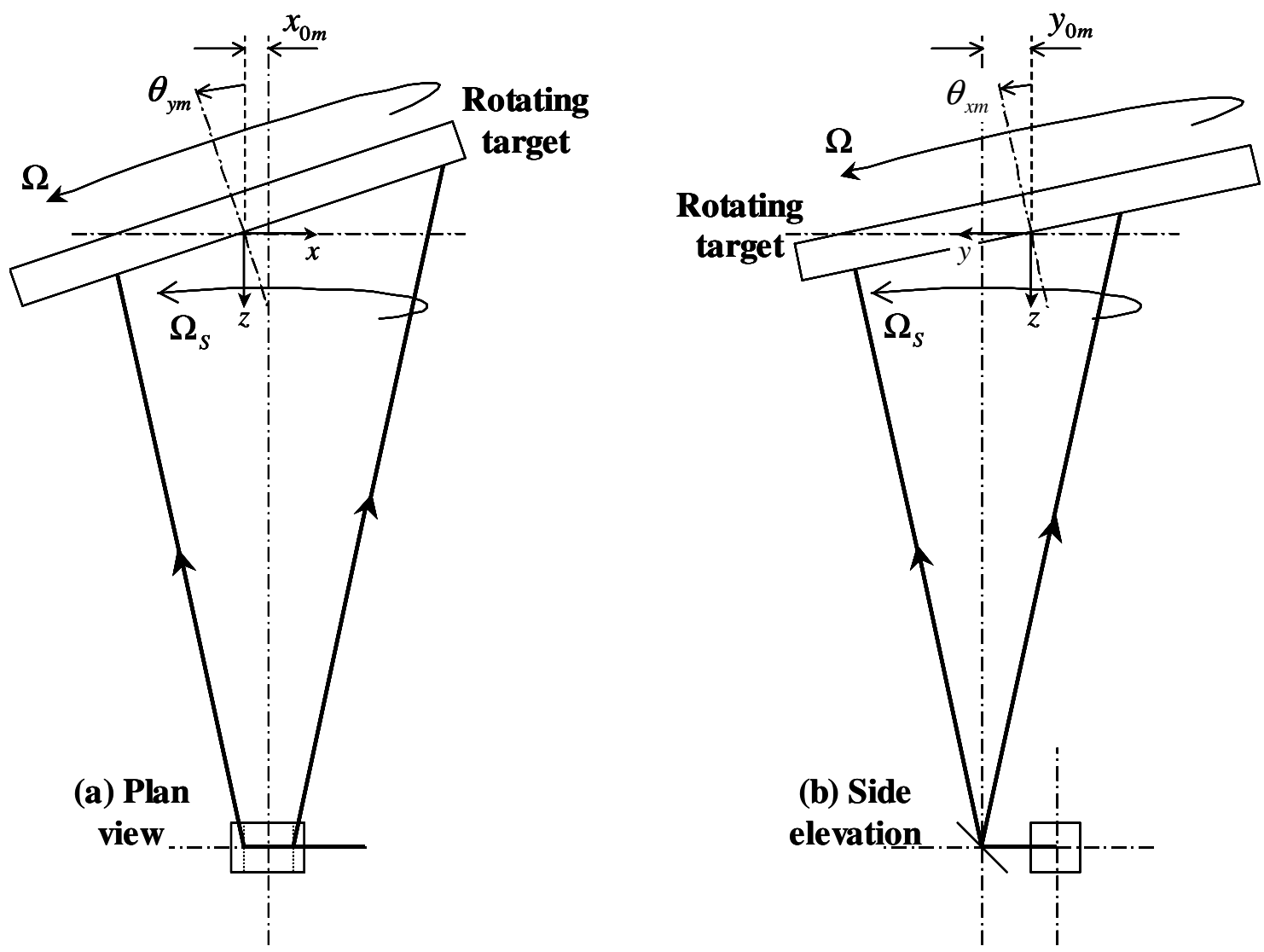

Figure 2 - Translational and angular misalignment between the scanning system and target rotation axes 


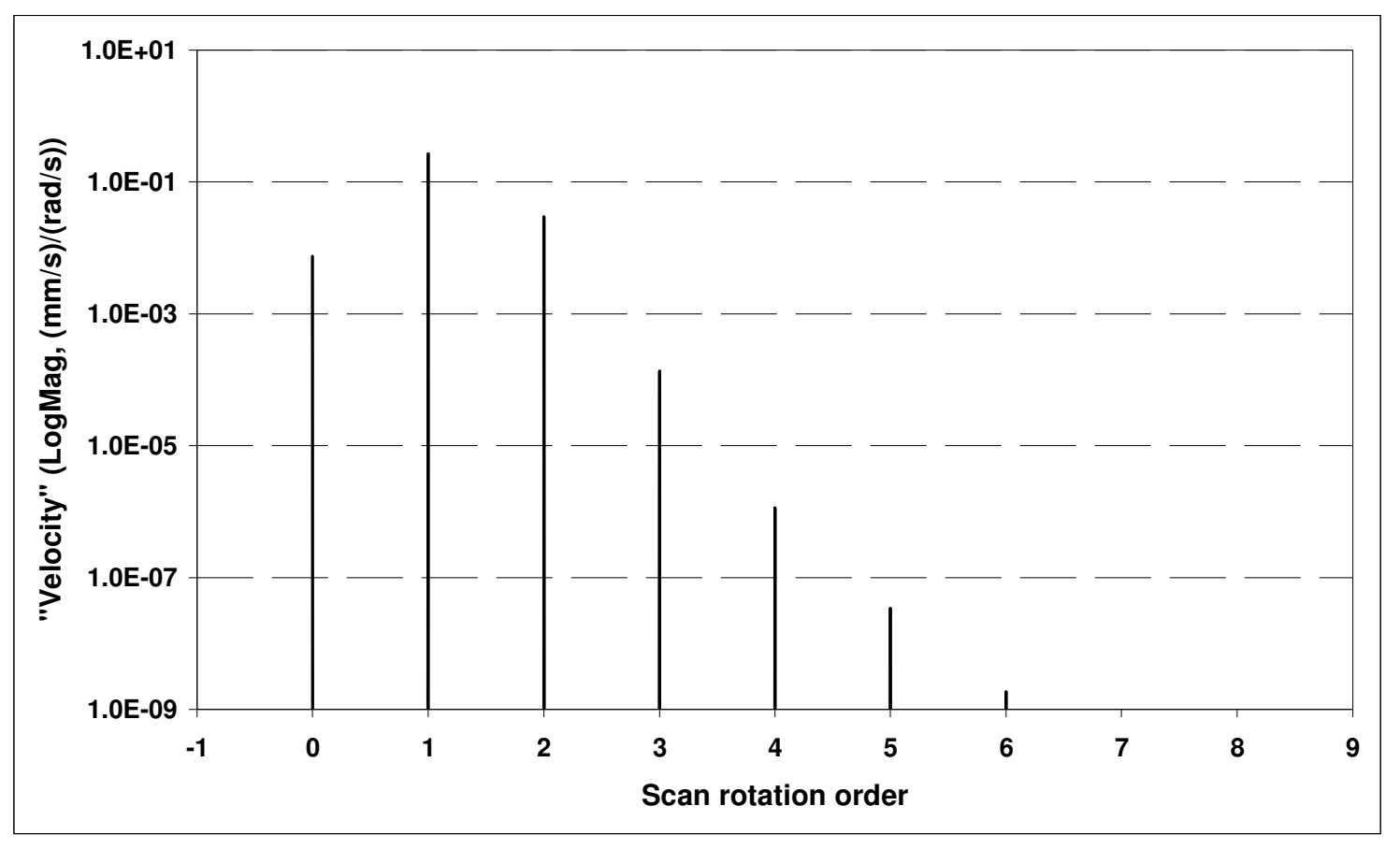

Figure 3 - Additional measurement components that occur due to misalignment between the dual mirror scanning system and target rotation axes $\left(r_{S}=100 \mathrm{~mm}, d_{S}=50 \mathrm{~mm}, z_{0}=1 \mathrm{~m}, x_{0 m}=y_{0 m}=2 \mathrm{~mm}\right.$ and $\left.\theta_{x m}=\theta_{y m}=15 \mathrm{mrad}\right)$
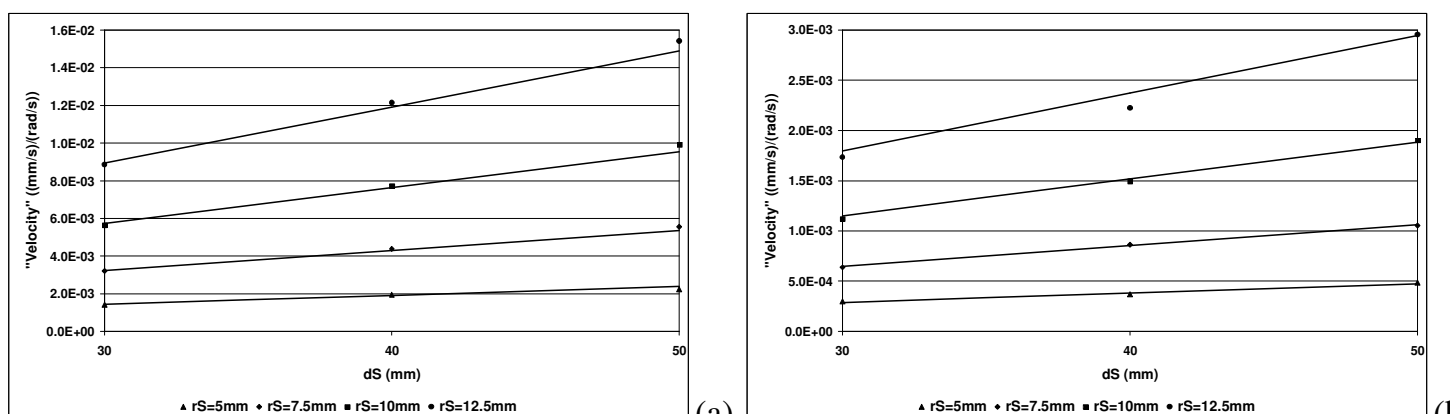

(a)

Figure 4 - Experimental validation of the additional measurement component at $2 x$ scan frequency for $z_{0}=0.512 \mathrm{~m}$ (a) and $z_{0}=1.127 \mathrm{~m} \mathrm{(b)}\left(\Omega_{S}=40 \pi \mathrm{rad} / \mathrm{s}\right)$ 

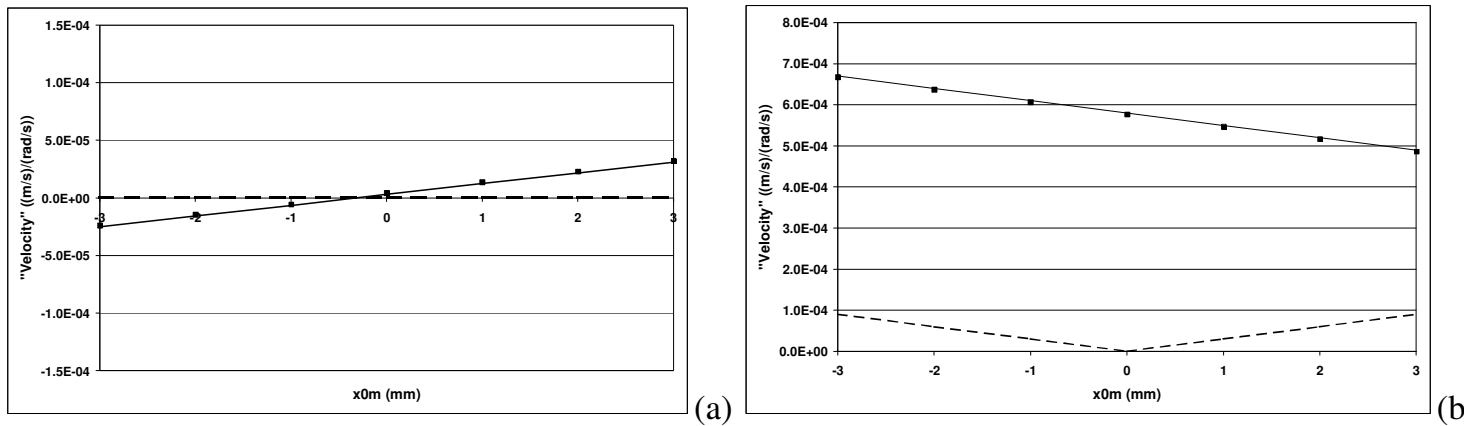

Figure 5 - Experimental validation of the additional measurement component at DC (a) and 1x scan frequency (b) for varying $x_{0 m}$ $\left(r_{S}=15 \mathrm{~mm}, d_{S}=50 \mathrm{~mm}, z_{0}=0.5 \mathrm{~m}, \Omega_{S}=40 \pi \mathrm{rad} / \mathrm{s}, y_{0 m}=0 \mathrm{~mm}\right.$ and $\left.\theta_{x m}=\theta_{y m}=0 \mathrm{mrad}\right)$, broken line $=$ initial prediction, solid line $=$ updated prediction
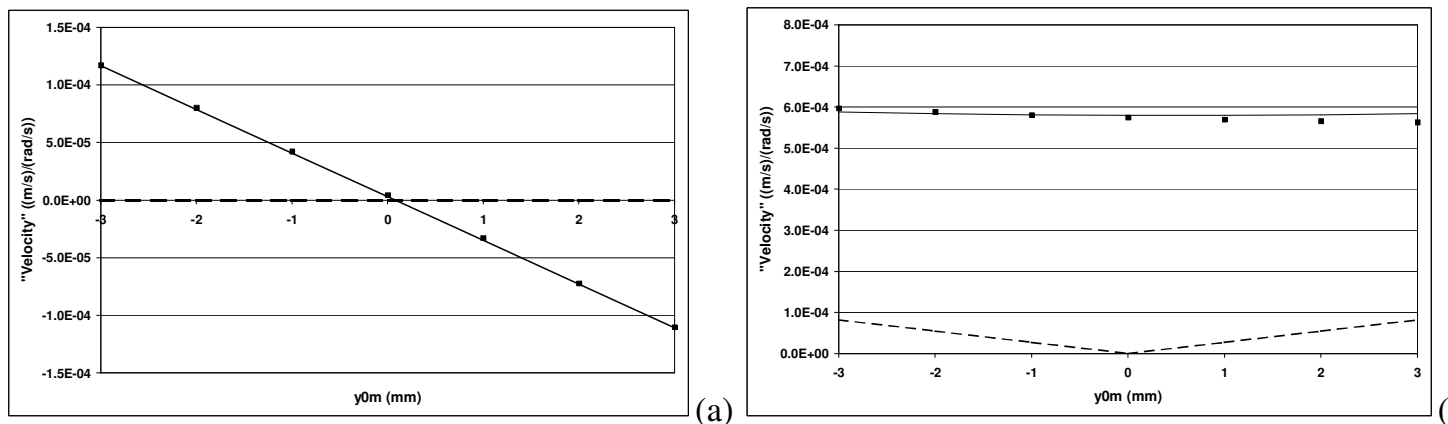

(a)

Figure 6 - Experimental validation of the additional measurement component at DC (a) and 1x scan frequency (b) for varying $y_{0 m}$ $\left(r_{S}=15 \mathrm{~mm}, d_{S}=50 \mathrm{~mm}, z_{0}=0.5 \mathrm{~m}, \Omega_{S}=40 \pi \mathrm{rad} / \mathrm{s}, y_{0 m}=0 \mathrm{~mm}\right.$ and $\left.\theta_{x m}=\theta_{y m}=0 \mathrm{mrad}\right)$, broken line $=$ initial prediction, solid line $=$ updated prediction
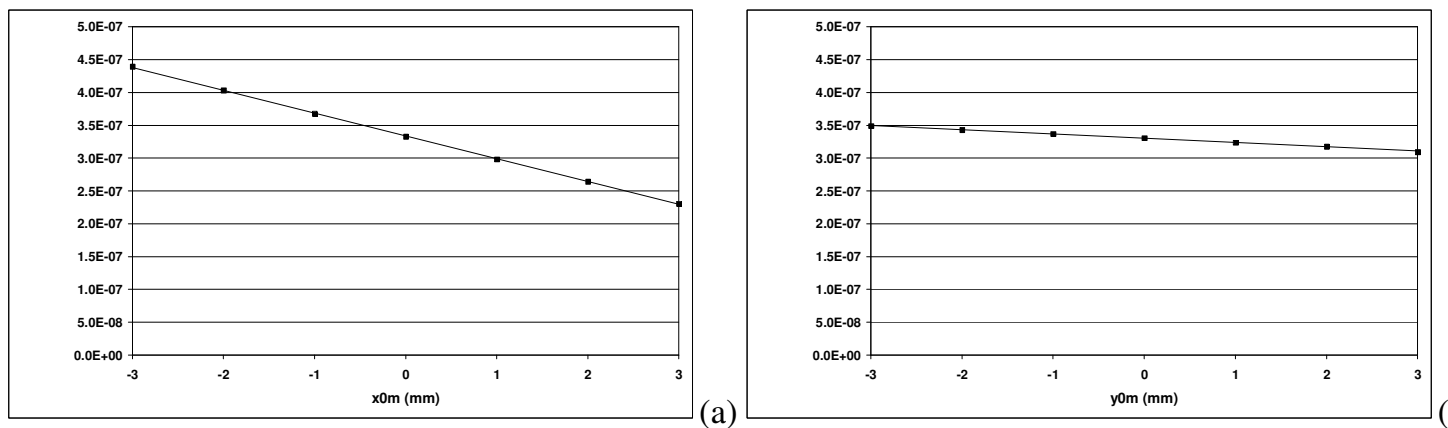

Figure 7 - Initial unknown misalignment analysis using variable $x_{0 m}$ (a) and variable $y_{0 m}(\mathrm{~b})$ 


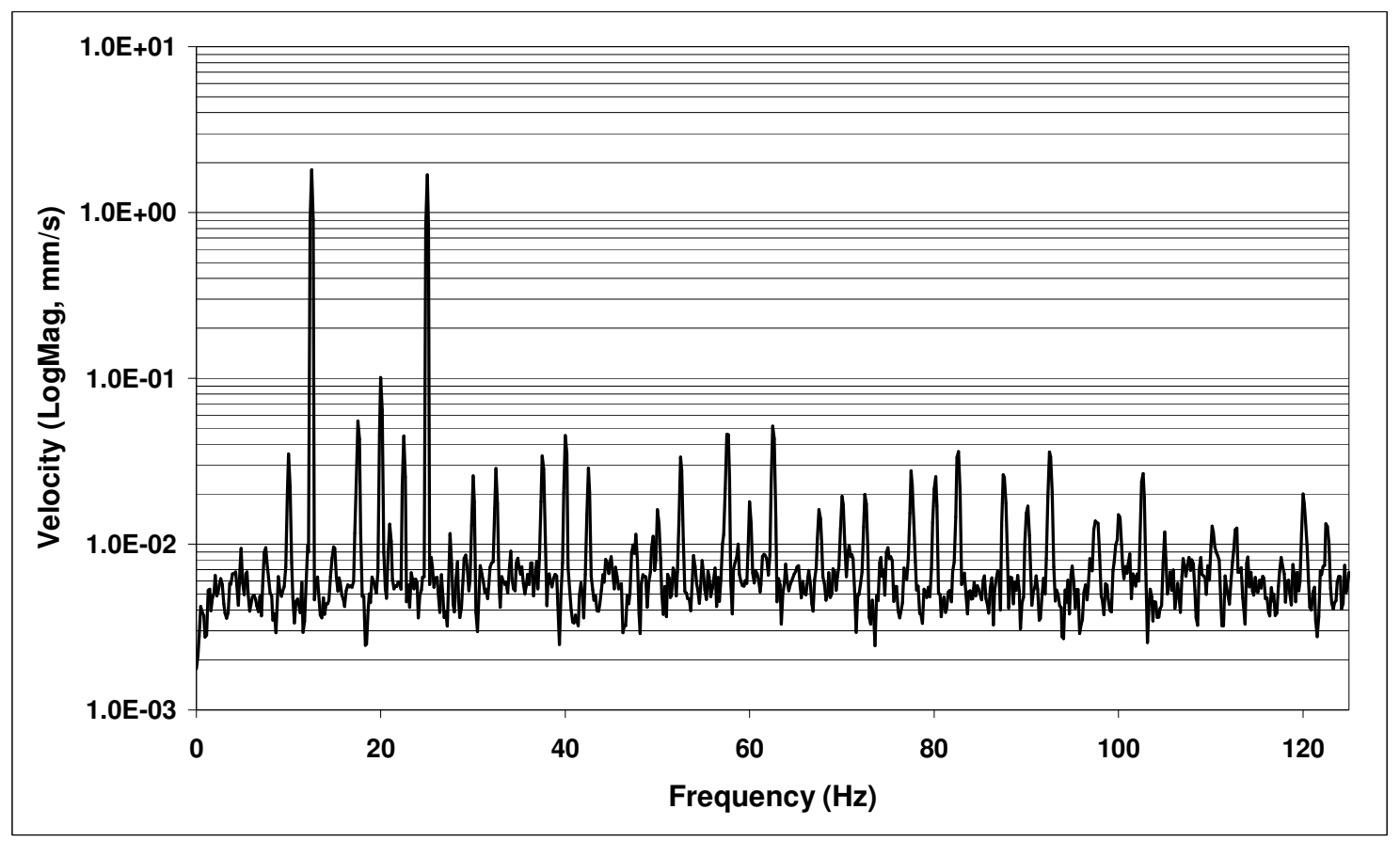

Figure 8 - Velocity measured by a circular scanning Laser Vibrometer on a rotating, non-vibrating target $\left(r_{S}=50 \mathrm{~mm}, d_{S}=50 \mathrm{~mm}, z_{0}=250 \mathrm{~mm}, \Omega_{S}=25 \mathrm{rad} / \mathrm{s}, \Omega \approx 20 \pi \mathrm{rad} / \mathrm{s}\right.$, arbitrary misalignment)

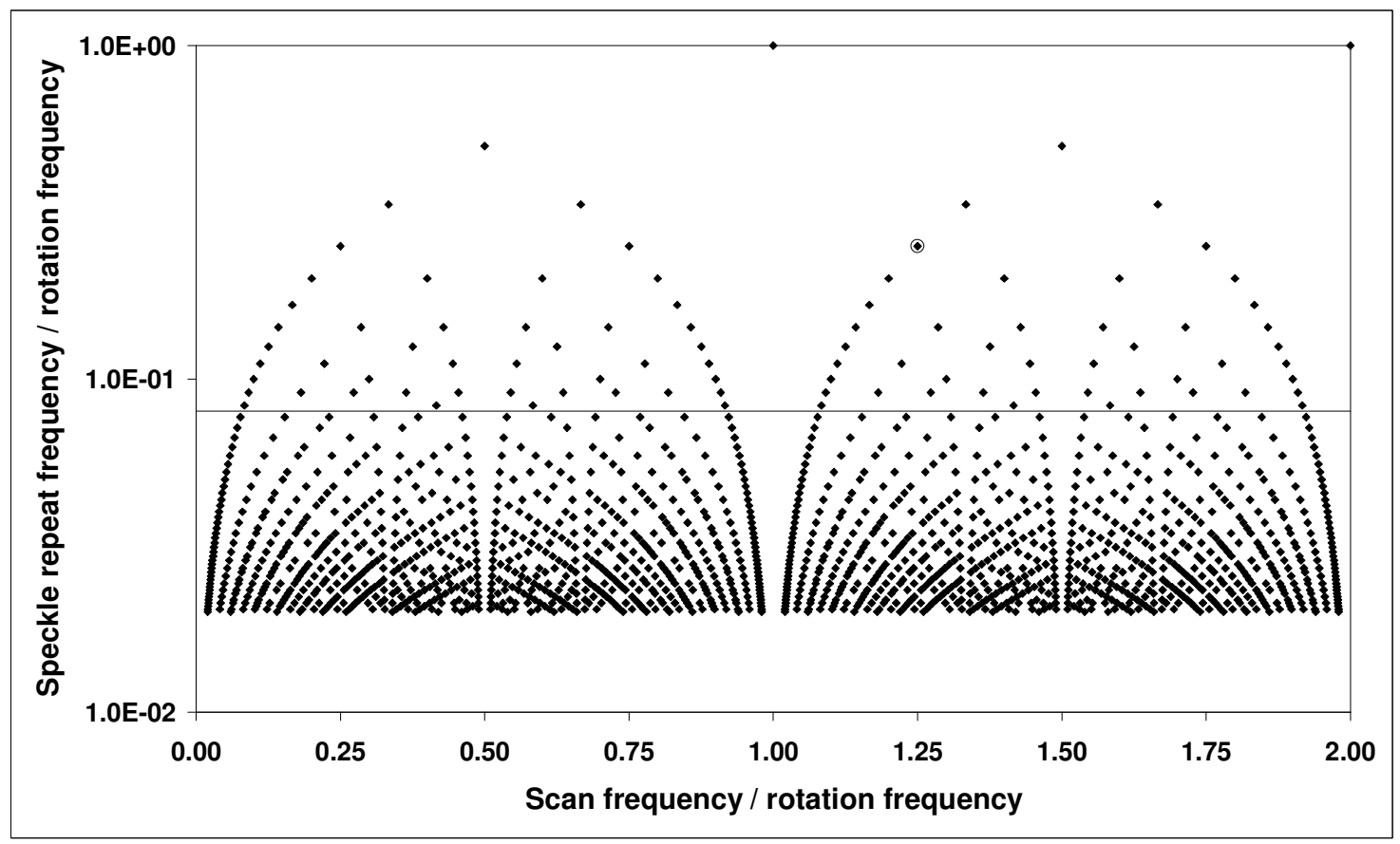

Figure 9 - Speckle repeat map 


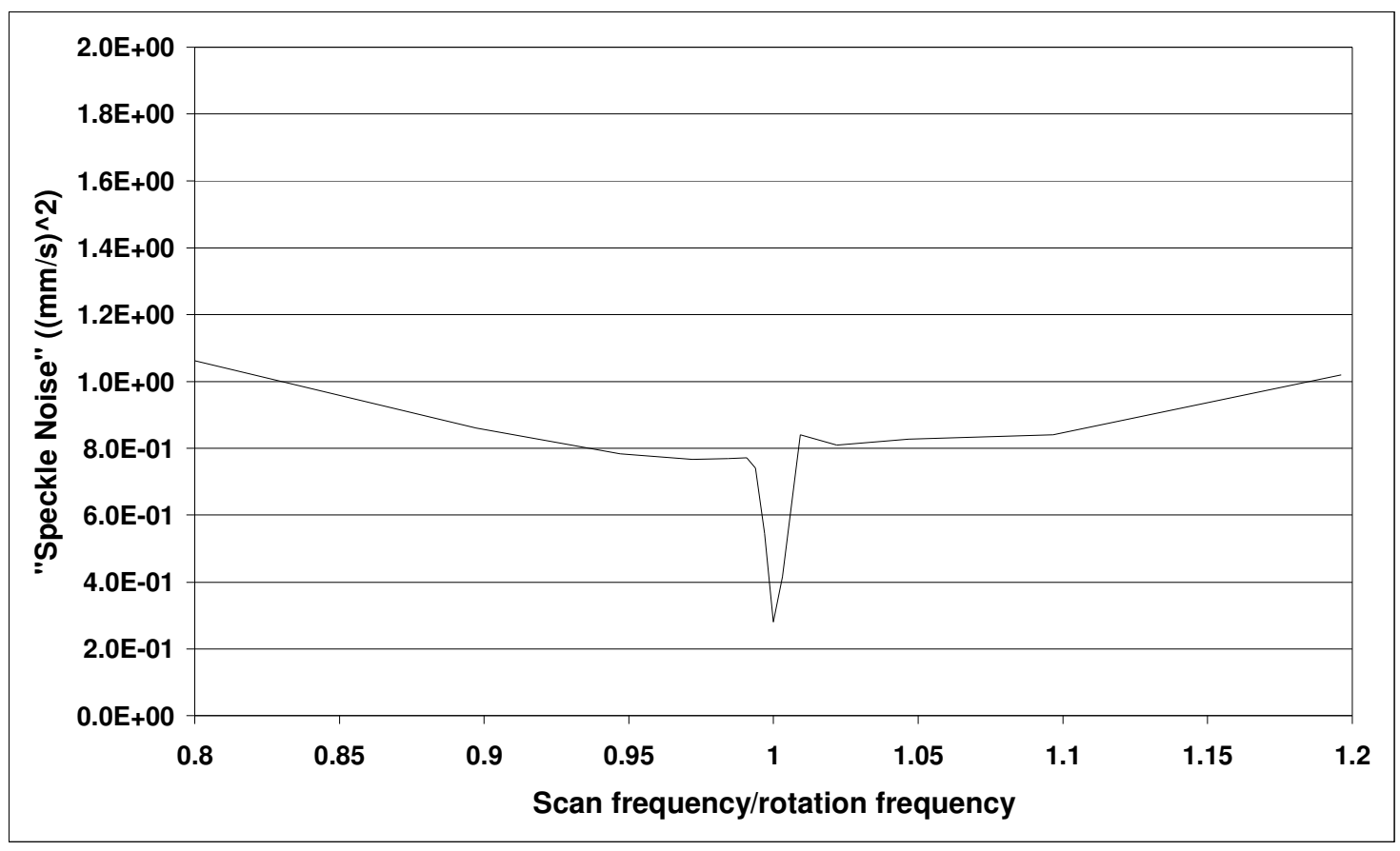

Figure 10 - Speckle noise in circular scanning Laser Vibrometer measurements on rotating targets 


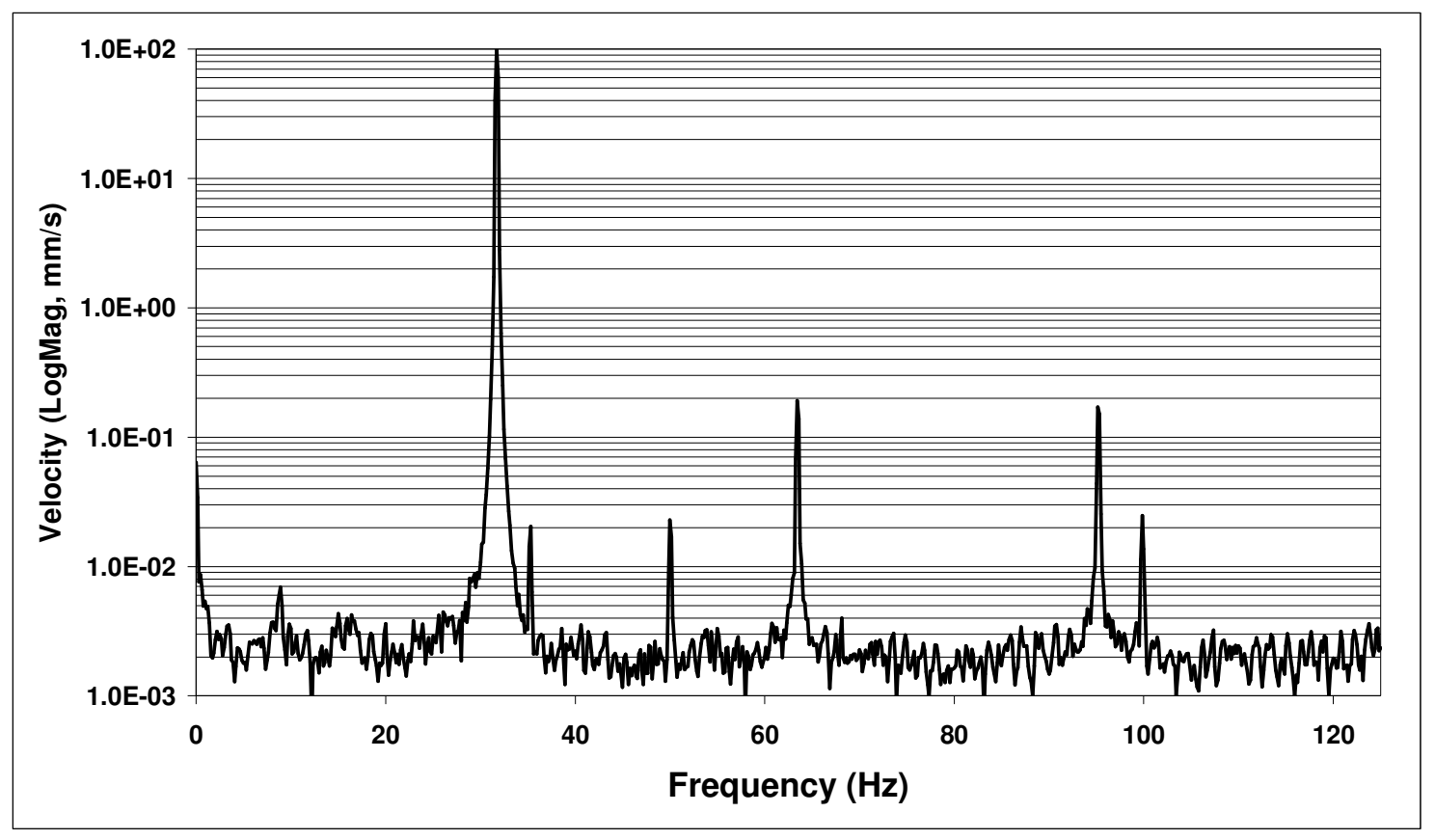

Figure 11 - Velocity measured by a Laser Vibrometer on a non-rotating blade undergoing $32.5 \mathrm{~Hz}, 100 \mathrm{~mm} / \mathrm{s}$ (nominal) vibration

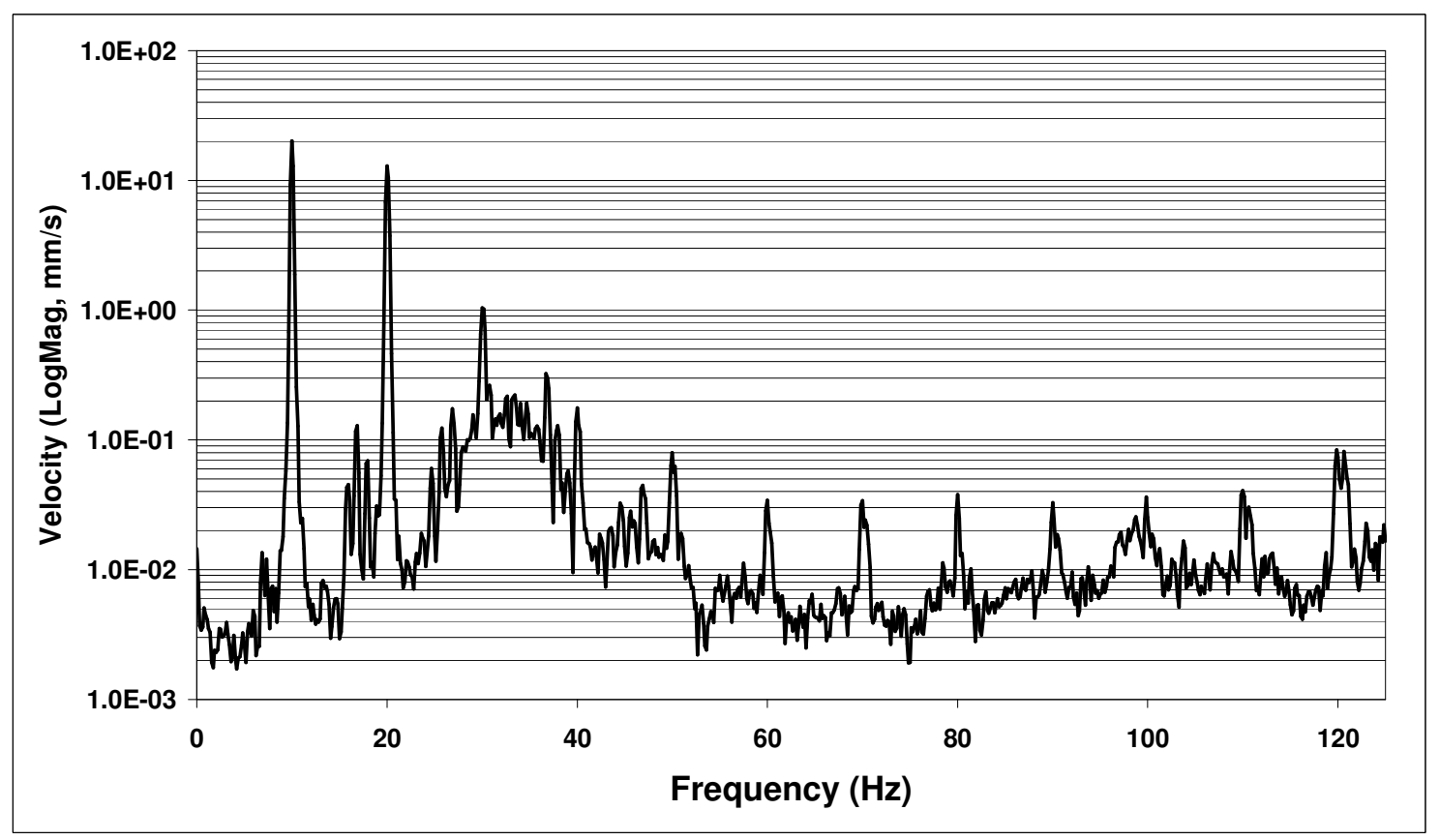

Figure 12 - Velocity measured by a circular tracking Laser Vibrometer on a (nominally) non-vibrating, rotating blade

( $r_{S}=100 \mathrm{~mm}, d_{S}=50 \mathrm{~mm}, z_{0}=1 \mathrm{~m}, \Omega_{S}=\Omega \approx 20 \pi \mathrm{rad} / \mathrm{s}$, arbitrary misalignment $)$ 


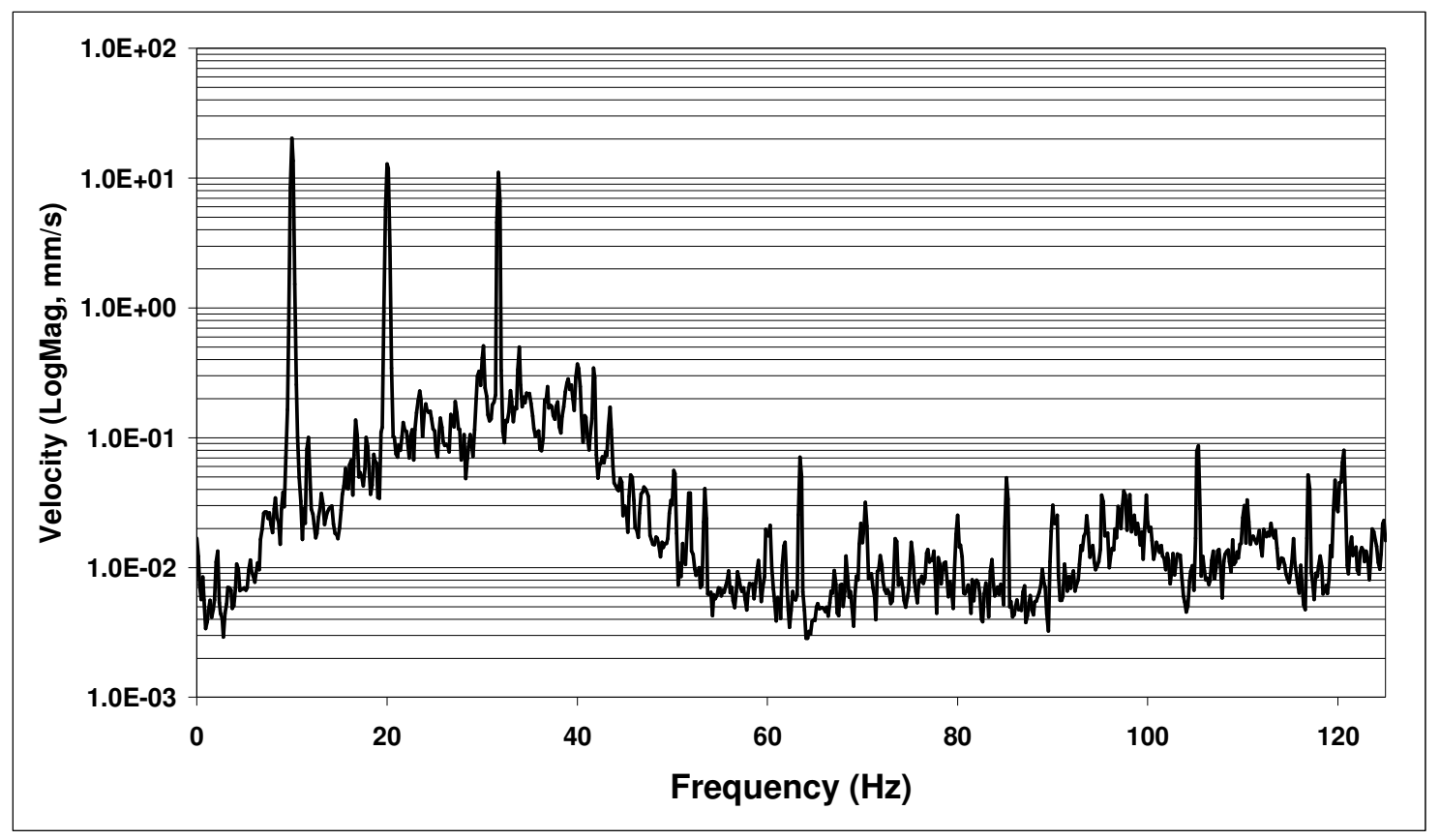

Figure 13 - Velocity measured by a circular tracking Laser Vibrometer on a rotating blade undergoing $32.5 \mathrm{~Hz}, 10 \mathrm{~mm} / \mathrm{s}$ (nominal) vibration

( $r_{S}=100 \mathrm{~mm}, d_{S}=50 \mathrm{~mm}, z_{0}=1 \mathrm{~m}, \Omega_{S}=\Omega \approx 20 \pi \mathrm{rad} / \mathrm{s}$, arbitrary misalignment $)$

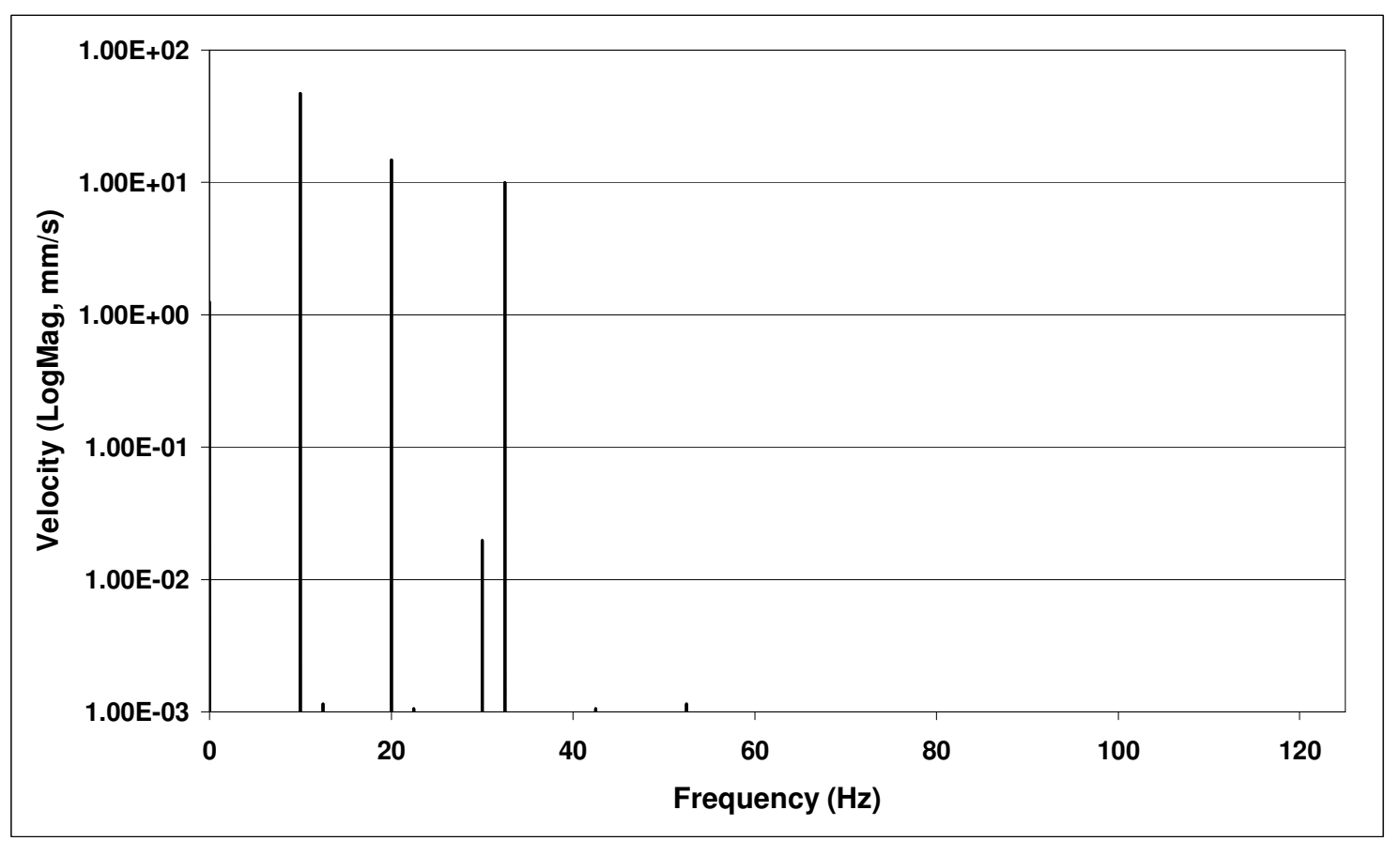

Figure 14 - Theoretical prediction of the velocity measured by a circular tracking Laser Vibrometer on a rotating target undergoing $32.5 \mathrm{~Hz}, 10 \mathrm{~mm} / \mathrm{s}$ axial vibration

$$
\begin{aligned}
\left(r_{S}=100 \mathrm{~mm}, d_{S}\right. & =50 \mathrm{~mm}, z_{0}=1 \mathrm{~m}, \Omega_{S}=\Omega \approx 20 \pi \mathrm{rad} / \mathrm{s}, \\
x_{0 m}=y_{0 m} & \left.=2 \mathrm{~mm} \text { and } \theta_{x m}=\theta_{y m}=5 \mathrm{mrad}\right)
\end{aligned}
$$



ERROR: undefined

OFFENDING COMMAND:

STACK: 